\title{
Progression and CSF Inflammation after Eradication of Oligoclonal Bands in an MS Patient Treated with Allogeneic Hematopoietic Cell Transplantation for Follicular Lymphoma
}

\author{
P. Braendstrup ${ }^{b} \quad$ A.R. Langkilde ${ }^{c} \quad$ K. Schreiber ${ }^{d}$ \\ M. Ravnborg ${ }^{\mathrm{e}} \quad$ F. Sellebjerg ${ }^{\mathrm{d}} \quad$ L. Vindel $\varnothing v^{\mathrm{a}, \mathrm{b}}$ \\ ${ }^{a}$ Allogeneic Hematopoietic Cell Transplantation Unit and ${ }^{\mathrm{b}}$ Allogeneic \\ Hematopoietic Cell Transplantation Laboratory, Department of Hematology, \\ 'Department of Radiology, and 'Sclerosis Clinic, Department of Neurology, \\ Copenhagen University Hospital Rigshospitalet, Copenhagen, and ${ }^{\mathrm{e}}$ Department \\ of Neurology, Odense University Hospital, Odense, Denmark
}

\section{Key Words}

Multiple sclerosis - Allogeneic hematopoietic cell transplantation - Disease-modifying therapies $\cdot$ CXCL13 $\cdot$ Matrix metalloproteinase- $9 \cdot$ Oligoclonal bands

\section{Abstract}

Background: Allogeneic hematopoietic cell transplantation (allo-HCT) has been proposed as treatment for multiple sclerosis (MS) and other autoimmune diseases.

Aims: To describe the effects of allo-HCT on the course of MS in a 49-year-old woman with longstanding progressive MS who was treated with allo-HCT for follicular lymphoma.

Methods: Non-myeloablative conditioning allo-HCT, examination for IgG oligoclonal bands and measurement of CXCL13 and matrix metalloproteinase-9 (MMP-9) concentration in the cerebrospinal fluid (CSF).

Results: Despite the disappearance of oligoclonal bands in CSF, disease progression and CSF inflammation was observed.

Conclusions: We hypothesize that CXCL13 and MMP-9 detected in CSF may reflect ongoing, pathogenic immune activation even after the eradication of intrathecal IgG synthesis. This suggests that progressive MS may depend more on innate than on adaptive immune activation. 


\section{Introduction}

Autologous hematopoietic cell transplantation (auto-HCT) treatment for multiple sclerosis (MS) involves high-dose chemotherapy (immunosuppression) that requires 'rescue' of the hematopoietic system with blood stem cells [1, 2]. In allogeneic hematopoietic cell transplantation (allo-HCT) the graft is obtained from an HLA-matched donor. In allo-HCT with myeloablative conditioning high-dose chemo-radiotherapy is given with the aim of eradicating the last tumor cell. Allo-HCT with non-myeloablative conditioning exploits the fact that the curative effect is mainly achieved by the ability of the donor immune system to eradicate recipient hematopoietic cells including malignant cells. The conditioning is mild and given only to prevent rejection of the donor cells [3].

\section{Case Presentation}

In July 2006 we treated a 49-year-old woman with MS with non-myeloablative conditioning allo-HCT for follicular lymphoma grade 1-2, stage IV. Prior to the transplant the lymphoma was brought into complete remission with chemotherapy. The graft consisted of peripheral blood stem cells from an HLA-identical sister (table 1).

Relapsing-remitting MS with onset in 1985 evolved into secondary progressive MS from 2000. She did not want immunomodulatory treatment at any time. She had no MS relapses from 2004 to 2006. Prior to HCT her expanded disability status scale (EDSS) score was 3.5 and her MS impairment scale (MSIS) score was 31 [4]. Following HCT she had new MRI disease activity and showed EDSS progression due to impairment of walking ability, which according to the patient partially resulted from pain after a deep venous thrombosis of the leg (table 1). Her MSIS score, which quantitates abnormalities on a standard neurological examination, also increased after HCT, but improved at later follow-up visits.

Before HCT she had IgG oligoclonal bands in cerebrospinal fluid (CSF) (fig. 1). B cell chemokine CXCL13 and matrix metalloproteinase-9 (MMP-9) levels in CSF, measured with commercially available ELISA kits (R\&D Systems, Abingdon, UK), were normal.

One-year post-transplant CSF examination was negative for oligoclonal bands, and MMP-9 levels were normal, but CXCL13 was detectable (fig. 1 and table 1). In April 2010 IgG oligoclonal bands remained absent, but CXCL13 was still detectable and the MMP-9 concentration had increased (table 1). CXCL13 concentrations were not measured in serum or plasma samples. Therefore, it cannot be excluded that CXCL13 in CSF might arise from plasma. Previous studies of CXCL 13 have, however, not detected CXCL13 in plasma in sufficiently high concentrations to have an impact on the CXCL13 concentrations measured in CSF [5]. We therefore consider it most likely that the CXCL13 measured in CSF arises from local synthesis. Furthermore, a polyclonal intrathecal IgG synthesis occurring in the absence of oligoclonal IgG synthesis is a theoretical possibility, but would be highly unusual in the setting of MS [6].

\section{Discussion}

The relationship between inflammation, demyelination and axonal pathology in MS is not resolved. Inflammation in relapsing-remitting MS is associated with demyelination and axonal loss. In progressive MS this relationship is less clear and it is thought that axonal degeneration may continue in the absence of overt inflammation [7], however, recent studies challenge this notion [8]. 
A handful of cases of MS patients treated with allo-HCT for malignant hematological disease have been published [1]. In two recent reports continued disease activity was observed after transplantation [9, 10]. In our patient allo-HCT was associated with the disappearance of IgG oligoclonal bands. This has also been observed in auto-HCT-treated MS patients [2]. However, serial CSF studies showed that even though IgG oligoclonal bands disappeared, CXCL13 and MMP-9 concentrations were increased. CXCL13 is expressed in perivascular infiltrates and within the brain parenchyma in MS, and CSF concentrations of CXCL13 are increased in secondary progressive MS $[5,11]$. Secondary progressive MS patients with a more severe disease course harbor lymphoid follicle-like structures in the meninges, and CXCL13 may be involved in the generation of these structures [12]. MMP-9 in CSF has also been associated with disease activity in MS [13]. In an autopsy study it was observed that although lymphocytes and plasma cells were almost absent after auto-HCT, there was ongoing microglia activation, demyelination, and axonal pathology [14]. We hypothesize that CXCL13 and MMP-9 detected in the CSF of our patient may reflect ongoing, pathogenic, immune activation associated with continuing tissue damage even after the eradication of intrathecal IgG synthesis. This is consistent with the notion that progressive MS may depend more on innate than on adaptive immune activation [8, 15].

\section{Disclosure Statement}

The authors declare that they have no conflicts of interest. 
Table 1. Overview of events, treatment, scores, paraclinical, and clinical findings

\begin{tabular}{|c|c|c|c|c|c|c|c|}
\hline Date & Clinical events & Treatment & $\begin{array}{l}\text { EDSS } \\
\text { score }\end{array}$ & $\begin{array}{l}\text { MSIS } \\
\text { score }\end{array}$ & MRI & $\begin{array}{l}\text { Oligoclonal CXCL13 } \\
\text { bands }\end{array}$ & MMP-9 \\
\hline 1985 & MS & & & & & & \\
\hline Nov. 2003 & follicular lymphoma & CVP, FC & & & & & \\
\hline June 2006 & CR of lymphoma & & 3.5 & 31 & $\begin{array}{l}>10 \mathrm{~T} 2 \text { brain lesions, } \\
\text { spinal MRI not done }\end{array}$ & $\begin{array}{l}\text { undetect- } \\
\text { able }\end{array}$ & undetectable \\
\hline July 2006 & Allo-HCT & FA, TBI, CsA, MMF1 & & & & & \\
\hline Aug. 2006 & & CsA, MMF & 3.5 & 30 & & & \\
\hline Jan. 2007 & acute GVHD & MEP, CsA, MMF, Infliximab & & & & & \\
\hline April 2007 & & CsA, MMF, PSL & 4 & 39 & & & \\
\hline Sept. 2007 & & MEP/PSL, MMF, TAC & & & $\begin{array}{l}\text { several new brain lesions } \\
\text { and } 2 \text { spinal cord lesions }\end{array}$ & & \\
\hline Dec. 2007 & $\begin{array}{l}\text { acute GVHD resolved/ } \\
\text { full donor chimera }\end{array}$ & MMF, TAC, PSL & & & & & \\
\hline July 2008 & chronic GVHD & PSL, TAC, MMF & & & & & \\
\hline Aug. 2008 & & PSL, TAC, MMF & & & $\begin{array}{l}1 \text { new brain lesion; } \\
1 \text { spinal cord lesion } \\
\text { resolved }\end{array}$ & $27 \mathrm{pg} / \mathrm{ml}$ & undetectable \\
\hline Sept. 2008 & CR of lymphoma & PSL, TAC, MMF & & & & & \\
\hline Dec. 2008 & & PSL, TAC, MMF & 5 & 33 & & & \\
\hline April 2010 & & TAC & & & & $24 \mathrm{pg} / \mathrm{ml}$ & $1.71 \mathrm{ng} / \mathrm{ml}$ \\
\hline June 2010 & & TAC & & & no changes & & \\
\hline May 2011 & & TAC & 5.5 & 32 & & & \\
\hline
\end{tabular}

${ }^{1}$ The patient has been receiving immunoglobulin replacement therapy since July 2006 due to hypogammaglobulinemia.

MRI = Magnetic resonance imaging; CXCL13 = C-X-C motif chemokine 13 (undetectable, i.e., $<7.8 \mathrm{pg} / \mathrm{ml}$ in control subjects); MMP-9= matrix metalloproteinase-9 (undetectable, i.e., $<0.0312 \mathrm{ng} / \mathrm{ml}$ in control subjects); GVHD = graft-versus-host disease; $\mathrm{CR}=$ complete remission; $\mathrm{CVP}$ = cyclophosphamide, vincristine, prednisolone; FC = fludarabine, cyclophosphamide; FA = fludarabine; TBI = total body irradiation; $\mathrm{MEP}$ = methylprednisolone; $\mathrm{CsA}$ = cyclosporine $\mathrm{A} ; \mathrm{PSL}=$ prednisolone; $\mathrm{MMF}$ = mycophenolate mofetil; $\mathrm{TAC}$ = tacrolimus. 


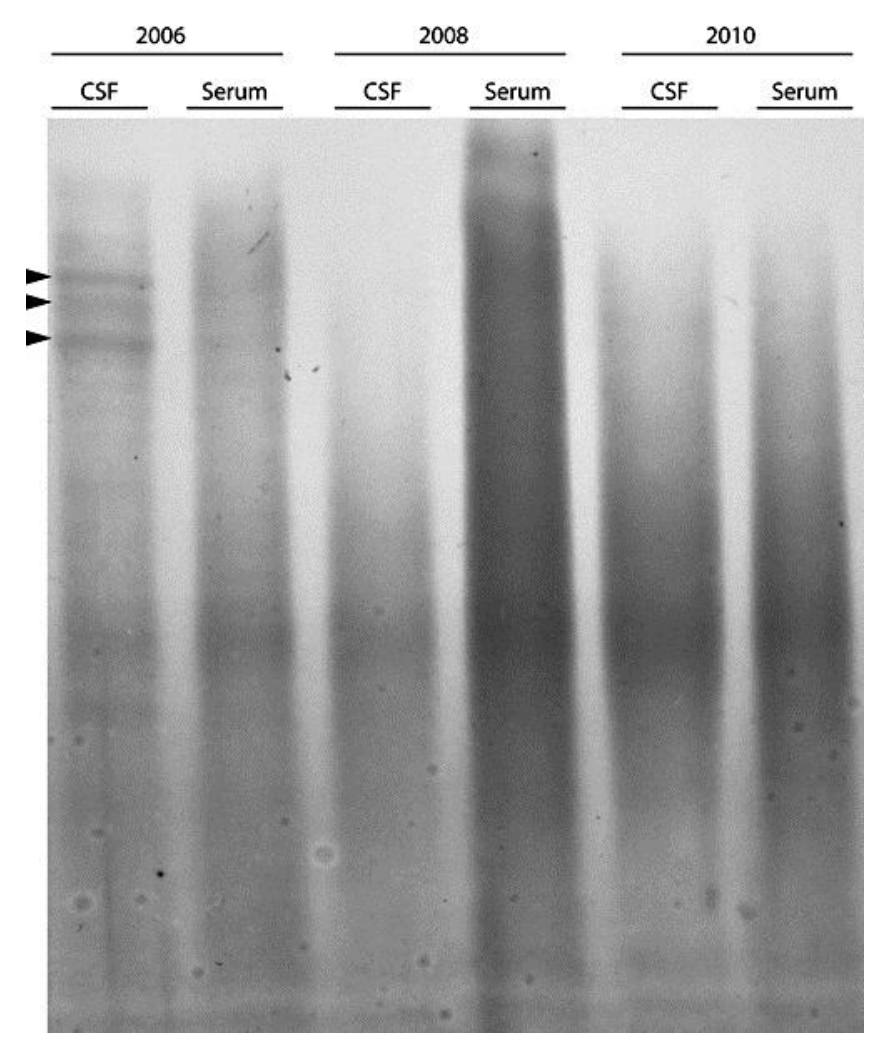

Fig. 1. Results of isoelectric focusing of IgG in CSF and serum from 2006 (before transplantation) and after transplantation in 2008 and 2010. The anode of the gel is at the bottom of the gel and the cathode is at the top of the gel. Oligoclonal bands are depicted by arrowheads. IgG oligoclonal bands were studied by isoelectric focusing in a commercially available isoelectric focusing gel followed by immunofixation with anti-IgG antibody and protein staining of fixed IgG-anti-IgG complexes in the gel (Hydragel 9 CSF isofocusing, Sebia Electrophoresis, Norcross, USA).

\section{References}

1 Van WB, Sprangers B, Dubois B, Waer M, Billiau AD: Autologous and allogeneic hematopoietic stem cell transplantation for multiple sclerosis: perspective on mechanisms of action. J Neuroimmunol 2008;197:89-98.

2 Nash RA, Bowen JD, McSweeney PA, Pavletic SZ, Maravilla KR, Park MS, et al: High-dose immunosuppressive therapy and autologous peripheral blood stem cell transplantation for severe multiple sclerosis. Blood 2003;102:2364-2372.

3 Gyurkocza B, Rezvani A, Storb RF: Allogeneic hematopoietic cell transplantation: the state of the art. Expert Rev Hematol 2010;3:285-299.

4 Ravnborg M, Blinkenberg M, Sellebjerg F, Ballegaard M, Larsen SH, Sorensen PS: Responsiveness of the Multiple Sclerosis Impairment Scale in comparison with the Expanded Disability Status Scale. Mult Scler 2005;11:81-84.

-5 Sellebjerg F, Bornsen L, Khademi M, Krakauer M, Olsson T, Frederiksen JL, et al: Increased cerebrospinal fluid concentrations of the chemokine CXCL13 in active MS. Neurology 2009;73:2003-2010.

6 Freedman MS, Thompson EJ, Deisenhammer F, Giovannoni G, Grimsley G, Keir G, et al: Recommended standard of cerebrospinal fluid analysis in the diagnosis of multiple sclerosis: a consensus statement. Arch Neurol 2005;62:865-870.

7 Compston A, Coles A: Multiple sclerosis. Lancet 2008;372:1502-1517. 
-8 Frischer JM, Bramow S, Dal-Bianco A, Lucchinetti CF, Rauschka H, Schmidbauer M, et al: The relation between inflammation and neurodegeneration in multiple sclerosis brains. Brain 2009;132:1175-1189.

-9 Jeffery DR: Failure of allogeneic bone marrow transplantation to arrest disease activity in multiple sclerosis. Mult Scler 2007;13:1071-1075.

10 Lu JQ, Storek J, Metz L, Yong VW, Stevens AM, Nash RA, et al: Continued disease activity in a patient with multiple sclerosis after allogeneic hematopoietic cell transplantation. Arch Neurol 2009;66:116-120.

11 Krumbholz M, Theil D, Cepok S, Hemmer B, Kivisakk P, Ransohoff RM, et al: Chemokines in multiple sclerosis: CXCL12 and CXCL13 up-regulation is differentially linked to CNS immune cell recruitment. Brain 2006;129:200-211.

12 Magliozzi R, Howell O, Vora A, Serafini B, Nicholas R, Puopolo M, et al: Meningeal B-cell follicles in secondary progressive multiple sclerosis associate with early onset of disease and severe cortical pathology. Brain 2007;130:1089-1104.

13 Sellebjerg F, Sorensen TL: Chemokines and matrix metalloproteinase-9 in leukocyte recruitment to the central nervous system. Brain Res Bull 2003;61:347-355.

14 Metz I, Lucchinetti CF, Openshaw H, Garcia-Merino A, Lassmann H, Freedman MS, et al: Autologous haematopoietic stem cell transplantation fails to stop demyelination and neurodegeneration in multiple sclerosis. Brain 2007;130:1254-1262.

15 Weiner HL: The challenge of multiple sclerosis: how do we cure a chronic heterogeneous disease? Ann Neurol 2009;65:239-248. 